\title{
Fair Division of Time: Multi-layered Cake Cutting
}

\author{
Hadi Hosseini $^{1}$, Ayumi Igarashi ${ }^{2}$ and Andrew Searns ${ }^{1}$ \\ ${ }^{1}$ Rochester Institute of Technology, USA \\ ${ }^{2}$ National Institute of Informatics, Japan \\ hhvcs@rit.edu, ayumi_igarashi@nii.ac.jp, abs2157@ rit.edu
}

\begin{abstract}
We initiate the study of multi-layered cake cutting with the goal of fairly allocating multiple divisible resources (layers of a cake) among a set of agents. The key requirement is that each agent can only utilize a single resource at each time interval. Several real-life applications exhibit such restrictions on overlapping pieces, for example, assigning time intervals over multiple facilities and resources or assigning shifts to medical professionals. We investigate the existence and computation of envy-free and proportional allocations. We show that envyfree allocations that are both feasible and contiguous are guaranteed to exist for up to three agents with two types of preferences, when the number of layers is two. We further devise an algorithm for computing proportional allocations for any number of agents when the number of layers is factorable to three and/or some power of two.
\end{abstract}

\section{Introduction}

Consider a group of students who wish to use multiple college facilities such as a conference room and an exercise room over different periods of time. Each student has a preference over what facility to use at different time of the day: Alice prefers to set her meetings in the morning and exercise in the afternoon, whereas Bob prefers to start the day with exercising for a couple of hours and meet with his teammates in the conference room for the rest of the day.

The fair division literature has extensively studied the problem of dividing a heterogeneous divisible resource (aka a cake) among several agents who may have different preference over the various pieces of the cake [Steinhaus, 1948; Robertson and Webb, 1998; Brams et al., 2006]. These studies have resulted in a plethora of axiomatic and existence results [Barbanel, 2005; Moulin, 2004] as well as computational solutions [Procaccia, 2013; Aziz and Mackenzie, 2016b] under a variety of assumptions, and were successfully implemented in practice (see [Procaccia and Moulin, 2016; Brams and Taylor, 1996] for an overview). In the case of Alice and Bob, each facility represents a layer of the cake in a multi-layered cake cutting problem, and the question is how to allocate the time intervals (usage right) of the facilities according to their preferences in a fair manner.

One naive approach is to treat each cake independently and solve the problem through well-established cake-cutting techniques by performing a fair division on each layer separately. However, this approach has major drawbacks: First, the final outcome, although fair on each layer, may not necessarily be fair overall. Second, the allocation may not be feasible, i.e., it may assign two overlapping pieces (time intervals) to a single agent. In our example, Alice cannot simultaneously utilize the exercise room and the conference room at the same time if she receives overlapping intervals. Several other application domains exhibit similar structures over resources: assigning nurses to various wards and shifts, doctors to operation rooms, and research equipment to groups, to name a few.

In multi-layared cake cutting, each layer represents a divisible resource. Each agent has additive preferences over every disjoint (non-overlapping) intervals. A division of a multi-layered cake is feasible if no agent's share contain overlapping intervals, and is contiguous if each allocated piece of a layer is contiguous. There has been some recent work on dividing multiple cakes among agents [Cloutier et al., 2010; Lebert et al., 2013]. Yet, none of the previous work considered the division of multiple resources under feasibility and contiguity constraints. Therefore, in this setting we ask the following research question:

What fairness guarantees can be achieved under feasibility and contiguity constraints for various number of agents and layers?

\subsection{Our Results}

We initiate the study of the multi-layered cake cutting problem for allocating divisible resources, under contiguity and feasibility requirements. Our focus is on two fairness notions, envy-freeness and proportionality. Envy-freeness (EF) requires that each agent believes no other agent's share is better than its share of the cake. Proportionality (Prop) among $n$ agents requires that each agent receives a share that is valued at least $\frac{1}{n}$ of the value of the entire cake. For efficiency, we consider complete divisions with no leftover pieces.

Focusing on envy-free divisions, we show the existence of envy-free and complete allocations that are both feasible and contiguous for two-layered cakes and up to three agents with at most two types of preferences. These cases are particularly 


\begin{tabular}{llll}
\hline Agents $(n)$ & Layers $(m)$ & EF & Prop \\
\hline 2 & 2 & Thm. 1 & Thm. 7 \\
3 & 2 & Thm. 2 & Thm. 7 \\
$n \geq m$ & $2^{a}, a \in \mathbb{Z}_{+}$ & $?$ & Thm. 7 \\
$n \geq m$ & $2^{a} 3^{b}, a \in \mathbb{Z}_{+}, b \in\{0,1\}$ & $?$ & Thm. $6^{\diamond}$ \\
\hline
\end{tabular}

Table 1: The overview of our results. $\dagger$ assumes two types of agents' preferences. $\diamond$ indicates that existence holds without contiguity requirement. Note that when $m>n$, no complete and feasible (non-overlapping) solution exists.

appealing since many applications often deal with dividing a small number of resources among few agents (e.g. assigning meeting rooms). We then show that proportional complete allocations that are feasible exist for three agents and three layers and can be computed efficiently. Based on this, we extend this result to a more general case; specifically, we show that a proportional complete feasible allocation exists when the number of layers is factorable to a product of three and powers of two. We omit some proofs due to space constraints. ${ }^{1}$

\subsection{Related Work}

In recent years, cake cutting has received significant attention in artificial intelligence and economics as a metaphor for algorithmic approaches in achieving fairness in allocation of resources [Procaccia, 2013; Brânzei and Nisan, 2019; Kurokawa et al., 2013; Aziz and Mackenzie, 2016a]. Recent studies have focused on the fair division of resources when agents have requirements over multiple resources that must be simultaneously allocated in order to carry out certain tasks (e.g. CPU and RAM) [Ghodsi et al., 2011; Gutman and Nisan, 2012; Parkes et al., 2015]. The most relevant work to ours is the envy-free multi-cake fair division that considers dividing multiple cakes among agents with linked preferences over the cakes. Here, agents can simultaneously benefit from all allocated pieces with no constraints. They show that envy-free divisions with only few cuts exist for two agents and many cakes, as well as three agents and two cakes [Cloutier et al., 2010; Lebert et al., 2013; Nyman et al., 2020]. In contrast, a multi-layered cake cutting requires non-overlapping pieces. Thus, the generalized envy-freeness notion on multiple cakes [Cloutier et al., 2010] does not immediately imply envy-freeness in our setting and no longer induces a feasible division.

\section{Our Model}

Our setting includes a set of agents denoted by $N=[n]$, a set of layers denoted by $L=[m]$, where for a natural number $s \in \mathbb{N},[s]=\{1,2, \ldots, s\}$. Given two real numbers $x, y \in \mathbb{R}$, we write $[x, y]=\{z \in \mathbb{R} \mid x \leq z \leq y\}$ to denote an interval. We denote by $\mathbb{R}_{+}$(respectively $\mathbb{Z}_{+}$) the set of non-negative reals (respectively, integers) including 0 . A piece of cake is a finite set of disjoint subintervals of $[0,1]$. We say that a subinterval of $[0,1]$ is a contiguous piece of cake. An $m$ layered cake is denoted by $\mathcal{C}=\left(C_{j}\right)_{j \in L}$ where $C_{j} \subseteq[0,1]$ is

\footnotetext{
${ }^{1}$ All missing proofs as well as the generalization of Theorem 4 can be found in the extended version [Hosseini et al., 2020].
}

a contiguous piece for $j \in L$. We refer to each $j \in L$ as $j$-th layer and $C_{j}$ as $j$-th layered cake.

Each agent $i$ is endowed with a non-negative integrable density function $v_{i j}: C_{j} \rightarrow \mathbb{R}_{+}$. For a given piece of cake $X$ of $j$-th layer, $V_{i j}(X)$ denotes the value assigned to it by agent $i$, i.e., $V_{i j}(X)=\sum_{I \in X} \int_{x \in I} v_{i j}(x) d x$. We assume that the valuations are normalized over layers, i.e., $\sum_{j \in L} V_{i j}\left(C_{j}\right)=1$. A layered piece is a sequence $\mathcal{X}=\left(X_{j}\right)_{j \in L}$ of pieces of each layer $j \in L$; a layered piece is said to be contiguous if each $X_{j}$ is a contiguous piece of each layer. We assume valuation functions are additive on layers and write $V_{i}(\mathcal{X})=$ $\sum_{j \in L} V_{i j}\left(X_{j}\right)$.

A layered contiguous piece is said to be non-overlapping if no two pieces from different layers overlap, i.e, for any pair of distinct layers $j, j^{\prime} \in L$ and for any $I \in X_{j}$ and $I^{\prime} \in X_{j^{\prime}}$, $I \cap I^{\prime}=\emptyset$. For two layered pieces $\mathcal{X}$ and $\mathcal{X}^{\prime}$, we say that agent $i$ weakly prefers $\mathcal{X}$ to $\mathcal{X}^{\prime}$ if $V_{i}(\mathcal{X}) \geq V_{i}\left(\mathcal{X}^{\prime}\right)$.

A multi-allocation $\mathcal{A}=\left(\mathcal{A}_{1}, \mathcal{A}_{2}, \ldots, \mathcal{A}_{n}\right)$ is a partition of the $m$-layered cake $\mathcal{C}$ where each $\mathcal{A}_{i}=\left(A_{i j}\right)_{j \in L}$ is a layered piece of the cake allocated to agent $i$; we refer to each $\mathcal{A}_{i}$ as a bundle of $i$. For a multi-allocation $\mathcal{A}$ and $i \in N$, we write $V_{i}\left(\mathcal{A}_{i}\right)=\sum_{j \in L} V_{i j}\left(A_{i j}\right)$ to denote the value of agent $i$ for $\mathcal{A}_{i}$. A multi-allocation $\mathcal{A}$ is said to be

- contiguous if each $\mathcal{A}_{i}$ for $i \in N$ is contiguous;

- feasible if each $\mathcal{A}_{i}$ for $i \in N$ is non-overlapping.

We focus on complete multi-allocations where the entire cake must be allocated. Notice that some layers may be disjoint (see Figure 1), and the number of agents must exceed the number of layers, i.e. $n \geq m$; otherwise the multi-allocation will contain overlapping pieces. We illustrate our model in the following example.

Example 1 (Resource sharing). Suppose that there are three meeting rooms $r_{1}, r_{2}$, and $r_{3}$ with different capacities, and three researchers Alice, Bob, and Charlie. The first room is available all day, the second and the third rooms are only available in the morning and late afternoon, respectively (see Fig. 1). Each researcher has a preference over the access time to the shared rooms. For example, Alice wants to have a group meeting in the larger room in the morning and then have an individual meeting in the smaller one in the afternoon.

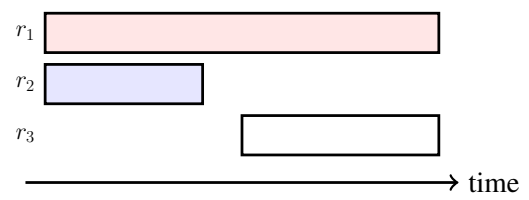

Figure 1: Example of a multi-layered cake. There are three meeting rooms $r_{1}, r_{2}$, and $r_{3}$ with different capacities, shared among several research groups.

Fairness. A multi-allocation is said to be envy-free if no agent envies the others, i.e., $V_{i}\left(\mathcal{A}_{i}\right) \geq V_{i}\left(\mathcal{A}_{i^{\prime}}\right)$ for any pair of agents $i, i^{\prime} \in N$. A multi-allocation is said to be proportional if each agent gets his proportional fair share, i.e., $V_{i}\left(\mathcal{A}_{i}\right) \geq \frac{1}{n}$ for any $i \in N$. The following implication, which is wellknown for the standard setting, holds in our setting as well. 
Lemma 1. An envy-free complete multi-allocation satisfies proportionality.

The $m$-layered cuts. In order to cut the layered cake while satisfying the non-overlapping constraint, we define a particular approach for partitioning the entire cake into diagonal pieces. Consider the $m$-layered cake $\mathcal{C}$ where $m$ is an even number. For each point $x$ of the interval $[0,1]$, we define

- $L R(x, \mathcal{C})=\left(\bigcup_{j=1}^{\frac{m}{2}} C_{j} \cap[0, x]\right) \cup\left(\bigcup_{j=\frac{m}{2}+1}^{m} C_{j} \cap[x, 1]\right)$;

- $R L(x, \mathcal{C})=\left(\bigcup_{j=1}^{\frac{m}{2}} C_{j} \cap[x, 1]\right) \cup\left(\bigcup_{j=\frac{m}{2}+1}^{m} C_{j} \cap[0, x]\right)$. $L R(x, \mathcal{C})$ consists of the top-half subintervals of points left of $x$ and the lower-half subintervals of points right of $x$; similarly, $R L(x, \mathcal{C})$ consists of the top-half subintervals of points right of $x$ and the lower-half subintervals of points left of $x$ (Fig. $2)$. We abuse the notation and write $L R(x)=L R(x, \mathcal{C})$ and $R L(x)=R L(x, \mathcal{C})$ if $\mathcal{C}$ is clear from the context.
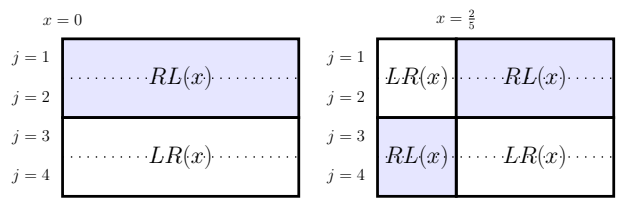

Figure 2: Examples of the partitions induced by $x=0$ and $x=\frac{2}{5}$ for a four-layered cake.

Computational model. Following the standard RobertsonWebb Model [Robertson and Webb, 1998], we introduce two types of queries: those for a cake on each layer (called a short knife) and those for the entire cake (called a long knife).

Short knife. Short eval query: given an interval $[x, y]$ of the $j$-th layered cake $C_{j}, \operatorname{eval}_{j}(i, x, y)$ asks agent $i$ for its value $[x, y]$, i.e., $V_{i j}([x, y])$. Short cut query: given a point $x$ and $r \in[0,1]$, cut $_{j}(i, x, r)$ asks agent $i$ for the minimum point $y$ such that $V_{i j}([x, y])=r$.

Long knife. Long eval query: given a point $x, \operatorname{eval}(i, x)$ asks agent $i$ for its value $L R(x)$, i.e., $V_{i}(L R(x))$. Long cut query: given $r \in[0,1], \operatorname{cut}(i, r)$ asks agent $i$ for the minimum point $x$ such that $V_{i}(L R(x))=r$ if such point $x$ exists.

\section{Existence of a Switching Point}

We start by showing the existence of a point $x$ that equally divides the entire cake into two pairs of diagonal pieces, both for the individuals and for the majority; these will serve as a fundamental property in our problem. We say that $x \in$ $[0,1]$ is a switching point for agent $i$ over a layered cake $\mathcal{C}$ if $V_{i}(L R(x))=V_{i}(R L(x))$.

Lemma 2. Suppose that the number $m$ of layers is even. Take any $i \in N$. Let $r \in \mathbb{R}$ be such that $(i) V_{i}(L R(0)) \geq r$ and $V_{i}(R L(0)) \leq r$, or $($ ii $) V_{i}(L R(0)) \leq r$ and $V_{i}(R L(0)) \geq r$. Then, there exists a point $x \in[0,1]$ such that $i$ values $L R(x)$ exactly at $r$, i.e. $V_{i}(L R(x))=r$. In particular, a switching point for $i$ always exists.

Proof. Suppose that $V_{i}(L R(0)) \geq r$ and $V_{i}(R L(0)) \leq r$. Consider the function $f(x)=V_{i}(L R(x))$ for $x \in[0,1]$. Recall that $f(x)$ is a continuous function written as the sum of continuous functions: $f(x)=\sum_{j=1}^{\frac{m}{2}} V_{i j}\left(C_{j} \cap[0, x]\right)+$ $\sum_{j=\frac{m}{2}+1}^{m} V_{i j}\left(C_{j} \cap[x, 1]\right)$. Since $f(0) \geq r$ and $f(1) \leq r$, there is a point $x \in[0,1]$ with $f(x)=r$ by the intermediate value theorem, which proves the claim. Further, by taking $r=\frac{1}{2}$, the point $x$ where $V_{i}(L R(x))=\frac{1}{2}$ is a switching point for agent $i$.

We will generalize the notion of a switching point from the individual level to the majority. For layered contiguous pieces $\mathcal{I}$ and $\mathcal{I}^{\prime}$, we say that the majority weakly prefer $\mathcal{I}$ to $\mathcal{I}^{\prime}$ (denoted by $\mathcal{I} \succeq \mathcal{I}^{\prime}$ ) if there exists $S \subseteq N$ such that $|S| \geq\left\lceil\frac{n}{2}\right\rceil$ and each $i \in S$ weakly prefers $\mathcal{I}$ to $\mathcal{I}^{\prime}$. We say that $x \in[0,1]$ is a majority switching point over $\mathcal{C}$ if $L R(x) \stackrel{m}{\succeq} R L(x)$ and $R L(x) \succeq L R(x)$. The following lemma guarantees the existence of a majority switching point, for any even number of layers and any number of agents.

Lemma 3. Suppose that the number $m$ of layers is even. Then, there exists a majority switching point for any number $n \geq m$ of agents.

\section{Envy-free Multi-layered Cake Cutting}

Now we will look into the problem of obtaining complete envyfree multi-allocations, while satisfying non-overlapping constraints. When there is only one layer, it is known that an envyfree contiguous allocation exists for any number of agents under mild assumptions on agents' preferences [Stromquist, 1980; Su, 1999]. Given the contiguity and feasibility constraints, the question is whether it is possible to guarantee an envy-free division in the multi-layered cake-cutting model.

\subsection{Two Agents and Two Layers}

We answer the above question positively for a simple, yet important, case of two agents and two layers. The standard protocol that achieves envy-freeness for two agents is known as the cut-and-choose protocol: Alice divides the entire cake into two pieces of equal value. Bob selects his preferred piece over the two pieces, leaving the remainder for Alice.

We extend this protocol to the multi-layered cake cutting using the notion of a switching point. Alice first divides the layered cake into two diagonal pieces: one that includes the top left and lower right parts and another that includes the top right and lower left parts of the cake. Our version of the cut-and-choose protocol is specified as follows:

Cut-and-choose protocol for $n=2$ agents over a twolayered cake $\mathcal{C}$ :

Step 1. Alice selects her switching point $x$ over $\mathcal{C}$.

Step 2. Bob chooses a weakly preferred layered contiguous piece among $L R(x)$ and $R L(x)$.

Step 3. Alice receives the remaining piece.

\begin{tabular}{|l|l|}
\hline \multicolumn{2}{|c}{$x$} \\
\hline$L R(x)$ & $R L(x)$ \\
\hline$R L(x)$ & $L R(x)$ \\
\hline
\end{tabular}

Figure 3: Cut-and-Choose for two-layered cake 
Theorem 1. The cut-and-choose protocol yields a complete envy-free multi-allocation that is feasible and contiguous for two agents and a two-layered cake using $O(1)$ number of long eval and cut queries.

Proof. It is immediate to see that the protocol returns a complete multi-allocation where each agent is assigned to a nonoverlapping layered contiguous piece. The resulting allocation satisfies envy-freeness: Bob does not envy Alice since he chooses a preferred piece among $L R(x)$ and $R L(x)$. Alice does not envy Bob by the definition of a switching point.

As we noted in Section 2, the existence result for two agents does not extend beyond two layers: if there are at least three layers, there is no feasible multi-allocation that completely allocates the cake to two agents.

\subsection{Three Agents and Two Layers}

We move on to the case of three agents and two layers. We will design a variant of Stromquist's protocol that achieves envy-freeness for one-layered cake [Stromquist, 1980]: The referee moves two knives: a short knife and a long knife. The short knife moves from left to right over the top layer and gradually increases the left-most top piece (denoted by $Y$ ), while the long knife keeps pointing to the point $x$, which can partition the remaining cake into two diagonal pieces $L R(x)$ and $R L(x)$ in an envy-free manner. Each agent shouts when the left-most top piece $Y$ becomes at least as highly valuable as the preferred piece among $L R(x)$ and $R L(x)$. Some agent, say $s$, shouts eventually (before the left-most top piece becomes the top layer), assuming that there is at least one agent who weakly prefers the top layer to the bottom layer. We note that $x$ may be positioned left to $y$; see Figure 4 for some possibilities of the long knife's locations.

We will show that the above protocol works, for a special case when there are at most two types of preferences: In such cases, the majority switching points coincide with the switching points of an agent with the majority preference.

Lemma 4. Suppose that $m=2, n=3$, and there are two different agents $i, j \in N$ with the same valuation $V$. Then, $x$ is a majority switching point over $\mathcal{C}$ if and only if $x$ is a switching point for $i$.

An obvious implication of the above lemma is that when performing Stromquist's protocol, one can point out to a switching point of an individual, instead of a majority one. This allows the value of each piece to change continuously. For a given two-layered cake $\mathcal{C}$, we write $\mathcal{C}^{-y}=\left(C_{1}^{-y}, C_{2}\right)$ as a two-layered cake obtained from $\mathcal{C}$ where the first segment $[0, y]$ of the top layer is removed, i.e., $C_{1}^{-y}=C_{1} \backslash[0, y]$. For each majority switching point $x$ over $\mathcal{C}^{-y}$, we select three different agents $\ell(x), m(x)$, and $r(x)$ as follows: $\ell(x)$ is an agent who weakly prefers $L R\left(x, \mathcal{C}^{-y}\right)$ to $R L\left(x, \mathcal{C}^{-y}\right)$; $m(x)$ is an agent who is indifferent between $\operatorname{LR}\left(x, \mathcal{C}^{-y}\right)$ and $R L\left(x, \mathcal{C}^{-y}\right)$; and $r(x)$ and agent who weakly prefers $R L\left(x, \mathcal{C}^{-y}\right)$ to $L R\left(x, \mathcal{C}^{-y}\right)$.

Theorem 2. Suppose that $m=2$ and $n=3$. If there are two different agents with the same valuation, an envy-free complete multi-allocation that is feasible and contiguous exists.

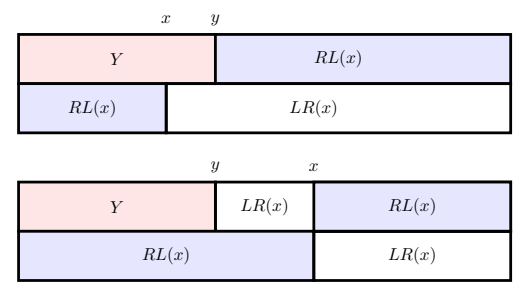

Figure 4: Moving knife protocol for three agents over a two-layered cake. Note that the position of $x$ may appear before $y$.

Proof. Assume w.l.o.g. that at least one agent prefers the top layer over the bottom layer. This means that such agent weakly prefers the top layer to any of the pieces $L R\left(z, \mathcal{C}^{-y}\right)$ and $R L\left(z, \mathcal{C}^{-y}\right)$ when $y=1$. Suppose that $i \in N$ is one of the two different agents with the same valuations. We design the following protocol for three agents over a two-layered cake:

Moving-knife protocol for $n=3$ agents over a twolayered cake $\mathcal{C}$ : w.l.o.g. assume that at least one agent weakly prefers the top layer $(j=1)$ over the bottom layer $(j=2)$

Step 1. The referee continuously moves a short knife from the left-most point $(y=0)$ to the right-most point $(y=1)$ over the top layer, while continuously moving a long knife pointing to a switching point over $\mathcal{C}^{-y}$ for $i$. Let $y$ be the position of the short knife and $Y$ be the top layer piece to its left. Let $x$ be the position of the long knife.

Step 2. The referee stops moving the short knife when some agent $s$ shouts, i.e., $Y$ becomes at least as highly valuable as the preferred piece among $L R\left(x, \mathcal{C}^{-y}\right)$ and $R L\left(x, \mathcal{C}^{-y}\right)$. Step 3. We allocate the shouter $s$ to the left-most top piece $Y$ and partitions the rest into $L R\left(x, \mathcal{C}^{-y}\right)$ and $R L\left(x, \mathcal{C}^{-y}\right)$.

- If $s=\ell(x)$, then we allocate $L R\left(x, \mathcal{C}^{-y}\right)$ to $m(x)$ and $R L\left(x, \mathcal{C}^{-y}\right)$ to $r(x)$.

- If $s=m(x)$, then we allocate $L R\left(x, \mathcal{C}^{-y}\right)$ to $\ell(x)$ and $R L\left(x, \mathcal{C}^{-y}\right)$ to $r(x)$.

- If $s=r(x)$, then we allocate $L R\left(x, \mathcal{C}^{-y}\right)$ to $\ell(x)$ and $R L\left(x, \mathcal{C}^{-y}\right)$ to $m(x)$.

By our assumption, some agent eventually shouts and thus the protocol returns an allocation $\mathcal{A}$. Clearly, $\mathcal{A}$ is feasible, contiguous, and complete. Also, it is easy to see that the shouter $s$ who receives a bundle $Y$ does not envy the other two agents. The agents $i \neq s$ do not envy $s$ because the referee continuously moves both a short and a long knife. Finally, the agents $i \neq s$ do not envy each other by the definition of a majority switching point and by Lemma 4 .

In the general case, the existence question of contiguous and feasible envy-free multi-allocations deems to be challenging due to the non-monotonicity of valuations over diagonal pieces. ${ }^{2}$ However, when the contiguity requirement is relaxed, one can show the existence of envy-free feasible multi-allocations [Hosseini et al., 2020].

\footnotetext{
${ }^{2}$ See Section 6 for an extensive discussion.
} 


\section{Proportional Multi-layered Cake Cutting}

Focusing on a less demanding fairness notion, it turns out that a complete proportional multi-allocation that is feasible exists for a wider class of instances, i.e., when the number $m$ of layers is a product of three and some power of two, and the number $n$ of agents is at least $m$. Notably, we show that the problem can be decomposed into smaller instances when the number of agents is greater than the number of layers, or the number of layers is a power of two. Building up on the base cases of two and three layers, our algorithm recursively calls the same algorithm to decide on how to allocate the cake of the sub-problems. We first proceed by showing how to solve the base case: For three layers and three agents, a feasible proportional multi-allocation exists and can be efficiently computed.

Theorem 3. A proportional complete multi-allocation that is feasible exists for three layers and three agents and can be computed using $O(1)$ number of short eval queries and long eval and cut queries. Further, each bundle of the resulting multi-allocation includes at most two contiguous pieces within each layer.

We start by showing two auxiliary lemmata. We define a merge of two disjoint contiguous pieces $I_{j}$ and $I_{j^{\prime}}$ of layers $j$ and $j^{\prime}$ as replacing the $j$-th layered cake with the union $I_{j} \cup I_{j^{\prime}}$ and removing $j^{\prime}$-th layered cake. The merge of a finite sequence of mutually disjoint contiguous pieces $\left(I_{1}, \ldots, I_{k}\right)$ can be defined inductively: merge $\left(I_{1}, \ldots, I_{k-1}\right)$ and then apply the merge operation to the resulting outcome and $I_{k}$. Now we observe that if there are two disjoint layers, one can safely merge these layers and reduce the problem size.

Lemma 5. Suppose that $C_{j}$ and $C_{j^{\prime}}$ are two disjoint layers of a layered cake $\mathcal{C}$, and $\mathcal{C}^{\prime}$ is obtained from $\mathcal{C}$ by merging $C_{j}$ and $C_{j^{\prime}}$. Then, each non-overlapping contiguous layered piece of $\mathcal{C}^{\prime}$ is a non-overlapping contiguous layered piece of the original cake $\mathcal{C}$.

The above lemma can be generalized further: Let $\mathcal{C}$ be a $2 m$-layered cake and $x \in[0,1]$. We define a merge of $L R(x)=\left(S_{j}\right)_{j \in L}$ by merging the pair $\left(S_{j}, S_{j+m}\right)$ for each $j \in[m]$. A merge of $R L(x)$ can be defined analogously. Such operation still preserves both feasibility and contiguity.

Corollary 1. Let $\mathcal{C}$ be a $2 m$-layered cake and $x \in[0,1]$. Suppose that $\mathcal{C}^{\prime}$ is a m-layered cake obtained by merging $L R(x, \mathcal{C})$ or $R L(x, \mathcal{C})$. Then, each non-overlapping contiguous layered piece of $\mathcal{C}^{\prime}$ is a non-overlapping contiguous layered piece of the original cake $\mathcal{C}$.

Below, we show that each agent can divide the entire cake into $n$ equally valued layered pieces. A multi-allocation $\mathcal{A}$ is equitable if for each agent $i \in N, V_{i}\left(\mathcal{A}_{i}\right)=\frac{1}{n}$. We design a recursive algorithm that iteratively finds two layers for which one has value at most $\frac{1}{m}$ and at least $\frac{1}{m}$ and removes a pair of diagonal pieces of value exactly $\frac{1}{m}$ from the two layers.

Lemma 6. For any number $m$ of layers and any number $n=m$ of agents with the identical valuations, an equitable complete multi-allocation that is feasible and contiguous exists and can be found using $O\left(\mathrm{~m}^{2}\right)$ number of short eval queries and $O(m)$ number of long cut queries.
Proof. We denote by $V=V_{i}$ the valuation function for each agent $i \in N$. Consider the following recursive algorithm $\mathcal{D}$ that takes a subset $N^{\prime}$ of agents with $\left|N^{\prime}\right| \geq 1$, a $\left|L^{\prime}\right|$ layered cake $\mathcal{C}^{\prime}$, and a valuation profile $\left(V_{i}\right)_{i \in N^{\prime}}$, and returns an equitable complete multi-allocation of the layered cake to the agents. When $\left|L^{\prime}\right|=\left|N^{\prime}\right|=1$, then the algorithm allocates the entire cake to the single agent. Suppose that $\left|L^{\prime}\right|=\left|N^{\prime}\right| \geq 2$. The algorithm first finds a layer $j$ whose entire value is at most $\frac{1}{m}$ and another layer $j^{\prime}$ whose entire value is at least $\frac{1}{m}$. The algorithm $\mathcal{D}$ then finds a point $x \in$ $[0,1]$ where $V\left(S_{j} \cup S_{j^{\prime}}\right)=\frac{1}{m}$ for $S_{j}=C_{j} \cap[0, x]$ and $S_{j^{\prime}}=C_{j} \cap[x, 1]$; such point exists due to Lemma 2 . We allocate $S_{j} \cup S_{j^{\prime}}$ to one agent and apply $\mathcal{D}$ to the remaining cake $\mathcal{C}^{\prime \prime}$ with $\left|N^{\prime}\right|-1$ agents where $\mathcal{C}^{\prime \prime}$ is obtained from merging the remaining $j$-th layered cake $C_{j} \backslash S_{j}$ and the $j^{\prime}$-th layered cake $C_{j^{\prime}} \backslash S_{j^{\prime}}$. The correctness of the algorithm as well as the bound on the query complexity are immediate.

Equipped with Lemma 6, we will prove Theorem 3.

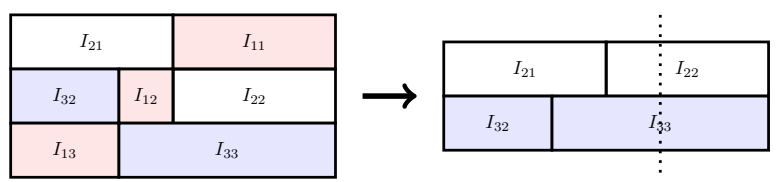

Figure 5: Protocol for proportionality for three agents and three layers. Alice divides the entire cake into three equally valued layered pieces $\mathcal{I}_{1}, \mathcal{I}_{2}$, and $\mathcal{I}_{3}$ (the left picture). Here, $\mathcal{I}_{i}=\left(I_{i j}\right)_{j=1,2,3}$ for each $i=1,2,3$ where $I_{23}=I_{31}=\emptyset$. After allocating $\mathcal{I}_{1}$ to either Alice or Charlie, the algorithm merges $\mathcal{I}_{2}$, and $\mathcal{I}_{3}$ and applies the cut-and-choose among the remaining agents (the right picture).

Proof of Thm. 3. Suppose there are three agents: Alice, Bob, and Charlie. Our procedure for achieving proportionality on a three-layered cake works as follows. See Figure 5 for an illustration.

A protocol for proportionality for $n=3$ agents over a three-layered cake $\mathcal{C}$ :

Step 1. Alice partitions the cake into three non-overlapping layered contiguous pieces $\mathcal{I}_{1}, \mathcal{I}_{2}$, and $\mathcal{I}_{3}$ which she considers of equal value, using the algorithm in the proof of Lemma 6. Step 2. Assume w.1.o.g. that $\mathcal{I}_{1}$ is the piece for which Bob has value at most $\frac{1}{3}$.

Step 3. Decide on the agent who is assigned to $\mathcal{I}_{1}$ depending on the preference of Charlie.

Step 3-1. If Charlie values $\mathcal{I}_{1}$ at least his proportional fair share $\frac{1}{3}$, then allocate it to him.

Step 3-2. Otherwise, allocate $\mathcal{I}_{1}$ to Alice.

Step 4. Merge all the disjoint contiguous pieces in $\mathcal{I}_{2}$ and $\mathcal{I}_{3}$, respectively, and create a two-layered cake $\mathcal{C}^{\prime}$ consisting of each merge of $\mathcal{I}_{2}$ and $\mathcal{I}_{3}$. Apply the cut-and-choose protocol to $\mathcal{C}^{\prime}$ among the remaining agents.

We will first show that the resulting multi-allocation $\mathcal{A}$ is proportional. Clearly, the agent who gets $\mathcal{I}_{1}$ has proportional fair share for his bundle. Further, each $i$ of the remaining agents have value at least $\frac{2}{3}$ for the remaining instance; thus, $V_{i}\left(\mathcal{A}_{i}\right)$ is at least $\frac{V_{i}\left(\mathcal{I}_{2}\right)+V_{i}\left(\mathcal{I}_{3}\right)}{2} \geq \frac{1}{3}$. Further, each bundle 
contains at most two contiguous pieces from each layer of $\mathcal{C}$ : If $\mathcal{A}_{i}=\mathcal{I}_{1}$, then $\mathcal{A}_{i}$ is contiguous. If $\mathcal{A}_{i}=L R\left(x, \mathcal{C}^{\prime}\right)$ for some $x \in R$, then each layer of $\mathcal{C}^{\prime}$ contains at most one contiguous piece of the original layers in $\mathcal{C}$; since $\mathcal{A}_{i}$ is contiguous with respect to $\mathcal{C}^{\prime}$, it contains at most two contiguous pieces of every distinct layer of $\mathcal{C}$. A similar argument applies to the case when $\mathcal{A}_{i}=R L\left(x, \mathcal{C}^{\prime}\right)$ for some $x \in R$. Finally, it can be easily verified that each bundle $\mathcal{A}_{i}$ is non-overlapping. This completes the proof.

We are now ready to prove that a proportional complete multi-allocation exists for any $n=m$ when $m$ is a product of some power of 2 and 3 . In essence, the existence of a majority switching point, as proved in Lemma 3, allows us to divide the problem into two instances. We will repeat this procedure until the number of layers of the subproblem becomes either 2 or 3 , for which we know the existence of a proportional, feasible multi-allocation by Theorem 1 and Theorem 3 .

Theorem 4. A proportional complete multi-allocation that is feasible exists for any number $m$ of layers and any number $n=m$ of agents where $m=2^{a} 3^{b}$ for some $a \in \mathbb{Z}_{+}$and $b \in\{0,1\}$.

Proof Sketch. We assume without loss of generality that each layer $C_{j}$ is the whole interval $[0,1]$ by just putting zero valuations on the part outside $C_{j}$. We design the following recursive algorithm $\mathcal{D}$ that takes a subset $N^{\prime}$ of agents with $\left|N^{\prime}\right| \geq 2$, a $\left|L^{\prime}\right|$-layered cake $\mathcal{C}^{\prime}$, and a valuation profile $\left(V_{i}\right)_{i \in N^{\prime}}$, and returns a proportional complete multi-allocation of the cake to the agents which is feasible. Suppose that $m=n$. If $m=n=1$, then we allocate the entire cake to the single agent. If $m=n=2$, we run the cut-and-choose algorithm as described in the proof of Theorem 1. If $m=n=3$, we run the procedure as described in the proof of Theorem 3. Now consider the case when $m=n=2^{a} 3^{b}$ for some integers $a \geq 1$ and $b \in\{0,1\}$. Then the algorithm finds a majority switching point $x$ over $\mathcal{C}^{\prime}$. We let $\mathcal{I}_{1}=L R(x)$ and $\mathcal{I}_{2}=R L(x)$. By definition of a majority switching point and the fact that $n$ is even, we can partition the set of agents $N^{\prime}$ into $N_{1}$ and $N_{2}$ where $N_{1}$ is the set of agents who weakly prefer $\mathcal{I}_{1}$ to $\mathcal{I}_{2}, N_{2}$ be the set of agents who weakly prefer $\mathcal{I}_{2}$ to $\mathcal{I}_{1}$, and $\left|N_{k}\right|=\frac{\left|N^{\prime}\right|}{2}$ for each $k=1,2$. We apply $\mathcal{D}$ to the merge of $\mathcal{I}_{k}$ with the agent set $N_{k}$ for each $k=1$, 2, respectively. One can prove by induction on $m$ that the complete multi-allocation $\mathcal{A}$ returned by $\mathcal{D}$ satisfies proportionality as well as feasibility.

It remains open whether a proportional contiguous multiallocation exists when the number of layers is three. A part of the reason is that our algorithm for finding an equitable multiallocation (Lemma 6) may not return a 'balanced' partition: The number of pieces contained in each layered piece may not be the same when the number of layers is odd. For example, one layered piece may contain pieces from three different layers while the other two parts may contain pieces from two different layers, as depicted in Figure 5. However, we are able to avoid this problem when the number of layers is a power of two: Indeed, in such cases, proportionality, contiguity, and feasibility are compatible with each other.
Theorem 5. A proportional complete multi-allocation that is feasible and contiguous exists for any number $m$ of layers and any number $n=m$ of agents where $m=2^{a}$ for some $a \in \mathbb{Z}_{+}$.

We will generalize the above theorems to the case when the number of agents is strictly greater than the number of layers. Intuitively, when $n>m$, then there is at least one layer whose sub-piece can be 'safely' allocated to some agent without violating the non-overlapping constraint.

Theorem 6. A proportional complete multi-allocation that is feasible exists for any number $m$ of layers and any number $n \geq m$ of agents where $m=2^{a} 3^{b}$ for some $a \in \mathbb{Z}_{+}$and $b \in\{0,1\}$.

The proof of the above theorem together with Theorem 5 immediately implies the following.

Theorem 7. A proportional complete multi-allocation that is feasible and contiguous exists for any number $m$ of layers and any number $n \geq m$ of agents where $m=2^{a}$ for some $a \in \mathbb{Z}_{+}$.

\section{Discussion}

We showed that an envy-free complete multi-allocation of a two-layered cake that is both contiguous and feasible exists for two or three agents with at most two types of preferences. An interesting open problem is whether such allocation also exists for any number of agents over a two-layered cake. One might expect that the Simmon-Su's technique [Su, 1999] using Sperner's Lemma can be adopted to our setting by considering all possible diagonal pieces. However, this approach may not work because multi-layered cake-cutting necessarily exhibits non-monotonicity in that the value of a pair of diagonal pieces may decrease when the knife moves from left to right.

For the case when the contiguity constraint is relaxed, one can show the existence of an envy-free feasible multiallocation, when each value density function $v_{i j}$ is continuous: We can reduce the problem to finding a 'perfect' allocation of a one-layered cake. The formal proof can be found in the extended version of the paper [Hosseini et al., 2020].

With respect to proportionality, one intriguing future direction is extending our positive results to any $m$, which requires careful consideration of contiguity and feasibility, which are often at odds with completeness. Lastly, the compatibility of the fairness notions with a more demanding efficiency requirement and studying its query complexity remain open in the multi-layered cake-cutting problem.

\section{Acknowledgements}

Hadi Hosseini was supported by the National Science Foundation (grant IIS-1850076). Ayumi Igarashi was supported by the KAKENHI Grant-in-Aid for JSPS Fellows no. 18J00997 and JST, ACT-X. The authors would like to thank Yuki Tamura for introducing the problem to us and for the fruitful discussions. The authors also acknowledge the helpful comments by the GAIW and IJCAI reviewers. We are grateful to an anonymous GAIW reviewer for the proof of the existence of an envy-free feasible multi-allocation in the general case. 


\section{References}

[Aziz and Mackenzie, 2016a] Haris Aziz and Simon Mackenzie. A discrete and bounded envy-free cake cutting protocol for any number of agents. In Proceedings of the 57th Symposium on Foundations of Computer Science (FOCS), pages 416-427, 2016.

[Aziz and Mackenzie, 2016b] Haris Aziz and Simon Mackenzie. A discrete and bounded envy-free cake cutting protocol for four agents. In Proceedings of the 48th Symposium on Foundations of Computer Science (FOCS), pages 454-464. ACM, 2016.

[Barbanel, 2005] Julius B Barbanel. The geometry of efficient fair division. Cambridge University Press, 2005.

[Brams and Taylor, 1996] Steven J. Brams and Alan D. Taylor. Fair Division: From Cake-Cutting to Dispute Resolution. Cambridge University Press, 1996.

[Brams et al., 2006] Steven J. Brams, Michal A. Jones, and Christian Klamler. Better ways to cut a cake. Notices of the American Mathematical Society, 53(11):1314-1321, 2006.

[Brânzei and Nisan, 2019] Simina Brânzei and Noam Nisan. Communication complexity of cake cutting. In Proceedings of the 20th ACM Conference on Economics and Computation (EC), pages 525-525. ACM, 2019.

[Cloutier et al., 2010] John Cloutier, Kathryn L Nyman, and Francis Edward Su. Two-player envy-free multi-cake division. Mathematical Social Sciences, 59(1):26-37, 2010.

[Ghodsi et al., 2011] Ali Ghodsi, Matei Zaharia, Benjamin Hindman, Andy Konwinski, Scott Shenker, and Ion Stoica. Dominant resource fairness: Fair allocation of multiple resource types. In Proceedings of the 8th USENIX Conference on Networked Systems Design and Implementation (NSD), pages 323-336. USENIX Association, 2011.

[Gutman and Nisan, 2012] Avital Gutman and Noam Nisan. Fair allocation without trade. In Proceedings of the 11th International Conference on Autonomous Agents and MultiAgent Systems (AAMAS), pages 719-728, 2012.

[Hosseini et al., 2020] Hadi Hosseini, Ayumi Igarashi, and Andrew Searns. Fair division of time: Multi-layered cake cutting. CoRR, abs/2004.13397, 2020.

[Kurokawa et al., 2013] David Kurokawa, John K Lai, and Ariel D Procaccia. How to cut a cake before the party ends. In Proceedings of the 27th AAAI Conference on Artificial Intelligence (AAAI), pages 555-561. AAAI Press, 2013.

[Lebert et al., 2013] Nicolas Lebert, Frédéric Meunier, and Quentin Carbonneaux. Envy-free two-player m-cake and three-player two-cake divisions. Operations Research Letters, 41(6):607-610, 2013.

[Moulin, 2004] Hervé Moulin. Fair division and collective welfare. MIT press, 2004.

[Nyman et al., 2020] Kathryn Nyman, Francis Edward Su, and Shira Zerbib. Fair division with multiple pieces. Discrete Applied Mathematics, 2020.
[Parkes et al., 2015] David C Parkes, Ariel D Procaccia, and Nisarg Shah. Beyond dominant resource fairness: Extensions, limitations, and indivisibilities. ACM Transactions on Economics and Computation (TEAC), 3:1-22, 2015.

[Procaccia and Moulin, 2016] Ariel D. Procaccia and Hervé Moulin. Cake Cutting Algorithms, pages 311 - 330. Cambridge University Press, 2016.

[Procaccia, 2013] Ariel D Procaccia. Cake cutting: not just child's play. Communications of the ACM, 56(7):78-87, 2013.

[Robertson and Webb, 1998] Jack Robertson and William Webb. Cake-cutting Algorithms: Be Fair if You Can. A. K. P.s, Ltd, 1998.

[Steinhaus, 1948] Hugo Steinhaus. The problem of fair division. Econometrica, 16(1):101-104, 1948.

[Stromquist, 1980] Walter Stromquist. How to cut a cake fairly. The American Mathematical Monthly, 87(8):640644, 1980.

[Su, 1999] Francis E. Su. Rental harmony: Sperner's lemma in fair division. The American Mathematical Monthly, 106(10):930-942, 1999. 